\title{
ON-LINE FLUORESCENT DNA SEQUENCING WITH A SCANNING OPTICAL SYSTEM
}

\author{
HIDEHIKO FUJII, SHIN NAKAMURA, MITSUHIRO HASHIMOTO, KATSUYA KASHIWAGI \\ and TOSHIHIRO WATANABE
}

Shimadzu Corporation, Biotechnology Instruments Department, 1 Nishinokyo-Kuwabaracho, Nakagyo-ku, Kyoto 604, Japan

\begin{abstract}
A laser induced fluorescence DNA sequencer is described. The excitation/objective optics are mounted on a mechanical scanner stage. The system allows the gel possible thinnest resulting in more than $\mathbf{4 5 0}$ mer peak resolution. Full two-dimensional data (gel width $\times$ time ladder pattern) are obtained, then can confirm up to gel entire raw data. All programs run on a multitasking operating system, which enables checking/processing obtained data whenever necessary even during electrophoresis. As a result, more than 500 bases sequencing was achieved from $0.5 \mu \mathrm{g} \mathrm{M13mp18}$ single stranded DNA template.
\end{abstract}

key words laser induced fluorescence, DNA sequencing, gel electrophoresis

\section{INTRODUCTION}

Recently several fluorescent DNA sequencers have been developed. Smith et al [1] reported the first fluorescent system, which used an electrophoresis capillary with 4 kinds of fluorescent label corresponding to 4 kinds of terminating base. However, mobility heterogeneities and spectroscopic sensitivity differences among the labelling dyes required a complicated preparation and data analysis. Prober et al [2] developed four fluorescent dyes whose excitation wavelengths are identical but whose emission spectra are different. They applied the dyes to dideoxy labelling in a one-lane one-template method. They also developed a slab gel detection system using the signal ratio between two fixed sensors detecting different wavelengths while the excitation laser beam was scanned by a galvano meter drive. However, because of detector number limitations, if the results were unexpected, it was difficult to know where the possible error occurred in a series of complicated processes.

In contrast with the above mentioned optics employing gel surface incidence, Ansorge et al [3] and Kambara et al [4] introduced incident light through the gel side wall and detected the emission along the normal direction of the gel. The configuration restricts the dimension of the gel relatively thick and/or narrow.

The authors see each prior apparatus as having some shortcoming for reliable sequencing. The first disadvantage is the difficulty in step by step data confirmation for unexpected results. The second major point is restriction of gel dimensions and/or the gel preparation method. In principle, the fluorescent method has some advantages in electrophoresis, such as, no glass preparation is needed and a large nucleotide length range capacity with a short migration length gel. The developmental philosophy for the apparatus described here (model DSQ-1, Shimadzu Corporation, Kyoto) is based on the above viowpoints.

\section{INSTRUMENTATION}

\subsection{OPTICAL CONFIGURATION}

Figure 1 shows our optical arrangement and the peripheral block diagram. Excitation light emitted from a 488nm $15 \mathrm{~mW}$ argon ion laser (LH in Fig.1) is changed in polarization from vertical to horizontal by two mirrors (M1 and M2) to eliminate Rayleigh scattering. The beam is made parallel to the scanning by M3 and is introduced to the mechanical scanner stage (SS). The incident beam (LB) is focused by lens LS1 (focal length $127 \mathrm{~mm}$ ) and deflected by M4 into acrylamide gel (AG) at an angle of $20^{\circ}$ to the gel surface. DNA tragment samples prepared according to Sanger's method [5] with FITC (fluorescein isothlocyanate) labelled primer are injected in wells $(W L)$ formed by a shark's tooth comb (SC) and migrate by electrophoresis down the gel (AG). 
Figure 1 Block Diagram of the System CL1-4:Control lines

ELC:Electrode cathode HVS:High voltage power supply LS1-3:Lenses MPU1-2:Micro processing units SL1-2:Signal lines Other notations in the text
ELA:Electrode anode MDL:Mirror drive linkage SDL:Scanner drive linkage

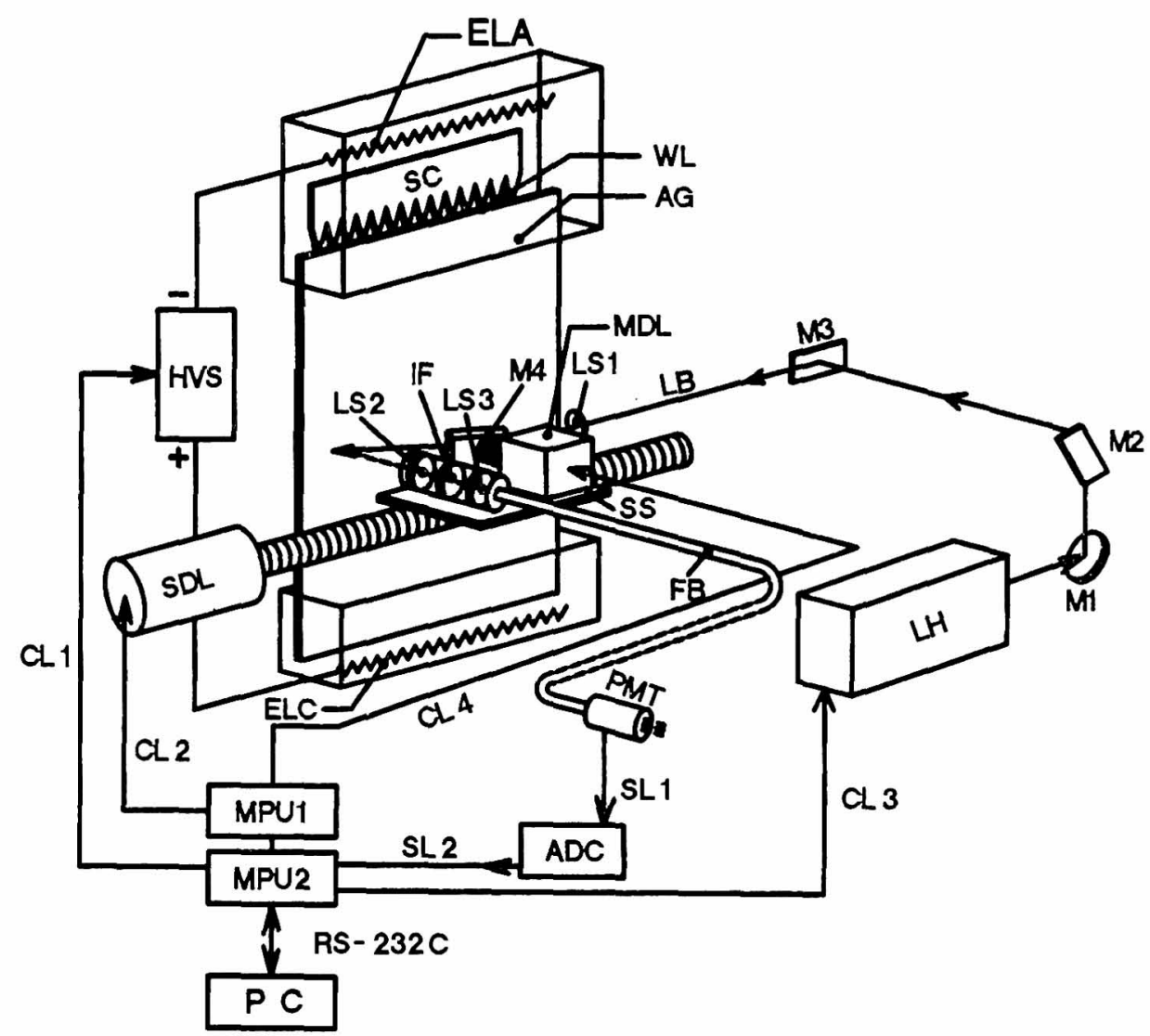

The emitted fluorescent light is collimated by an objective lens (LS2) of 0.45 numerical aperture and is then filtered by an interference filter IF (520nm : maximum emission wavelength of FITC) and focused by LS3 into an optical fiber bundle (FB) which is terminated with a photomultiplier tube (PMT) external to the scanner stage.

The scanner stage (SS) reciprocates every 5 seconds and covers $220 \mathrm{~mm}$ of gel width. During one scan total 512 points data are collected with 12 bit resolution (ADC : analog to digital converter) and all raw data are stored in the hard disk of an external personal computer (PC) through an RS-232C (9600 BPS) communication line.

The present system employs an automated system which continuously adjusts the observing optical axis with respect to the point of illumination on the gel. (Figure 2)

Figure 2 Objective Lens and Excitation Laser Beam

Arrow indicates the illuminated gel position, which is automatically adjusted to the focal point of the objective lens.

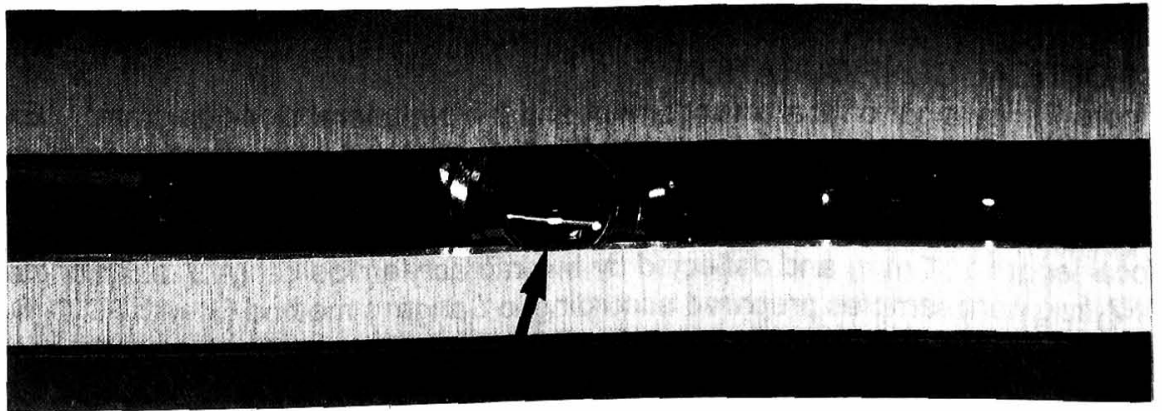




\subsection{DATA ANALYSIS}

Data analysis is performed by a 32 bit personal computer (PC-9801 series, NEC,Tokyo). The stored raw data of entire gel plate are refined to final sequence data as follows:

(Step 1)display as a ladder pattern (false colored fluorescent signal intensity) of all gel information.

(Step 2)lane definition by automated or manual method.

(Step 3)superimpose the defined lane onto the ladder pattern.

(Step 4)check or change the lane by user

(Step 5)get time signals of family 4 lanes.

(Step 6)noise removal from the time signals with a Fourier filter.

(Step 7)peak finding/ordering and smiling compensation.

(Step 8)store obtained sequence to file.

The data can be handled even during electrophoresis (multitasking on MS-Windows ${ }^{\text {mw }}$ Ver 3.0).

\section{EXPERIMENTAL PROCEDURE}

\subsection{SAMPLE PREPARATION}

(Step 1)(Annealing ) $0.4 \mathrm{pmol}(1 \mu \mathrm{g})$ of M13mp18 single strand DNA template and $1 \mathrm{pmol}(1 \mu \mathrm{l})$ FITC labelled 20 mer primer (both purchased from Wakunaga Pharmaceutical Co. Ltd) and $1.5 \mu \mathrm{l}$ of $5 \mathrm{X}$ sequencing reaction buffer are mixed and kept 10 minutes at $65{ }^{\circ} \mathrm{C}$ and are then allowed to cool to room temperature in $\mathbf{3 0}$ minutes.

(Step 2) $0.8 \mu$ l of 4 kind $(A, G, C, T)$ of d/dideoxy nucleotide mixture were pored separately into 4 vials.

(Step 3) (Extension) $2.2 \mu \mathrm{l}$ of mixture of $1 \mu \mathrm{l}$ of $0.1 \mathrm{M}$ dithiosleitol and $1 \mu \mathrm{l}$ of mixture of $3.5 \mu \mathrm{l}$ of enzyme dilution buffer and $1.5 \mu$ lof T7 DNA polymerase (purchased from Wakunaga Pharmaceutical Co.Ltd.) with annealed template/primer mixture above were added to each d/dNTP vial which were then heated at $37^{\circ} \mathrm{C}$ for 1 minute.

(Step 4) After step 3, the four vials were immediately put into ice. Then $2 \mu$ l of stop solution was added to each(final volume $5.5 \mu \mathrm{l}$ ), after which they were stored at $-20^{\circ} \mathrm{C}$.

(Step 5) Just before sampling, the 4 vials were heated at $95^{\circ} \mathrm{C}$ for 2 minutes and immediately plunged into ice, and then the contents injected $(2 \mu \mathrm{leach})$ to the electrophoresis wells. (Consequently, the loaded amount of template was less than $0.5 \mu \mathrm{g}$ ).

\subsection{ELECTROPHORESIS}

The electrophoresis gel was $260 \mathrm{~mm}$ wide, $0.25 \mathrm{~mm}$ thick, $27 \mathrm{~cm}$ of migration width from the loading well to the detection line. It contained $5 \%$ of acrylamide (includes $5 \%$ of BIS), $8 \mathrm{M}$ urea, $0.05 \%$ of TEMED and $0.05 \%(\mathrm{~W} / \mathrm{V})$ of APS in electrophoresis buffer as final. The electrophoresis buffer contained $90 \mathrm{mM}$ tris-base and $90 \mathrm{mM}$ boric acid and $2.5 \mathrm{mM}$ of EDTA. The same buffer was also pored into the two electrode reservoirs (see Figure 1). All electrophoresis reagents were purchased from Wako Pure Chemicals Inc, Osaka. The gel had been left at room temperature for more than 6 hours after preparation. Sample electrophoresis was performed for $12 . .5$ hour under $20 \mathrm{~W}$ constant power at $45^{\circ} \mathrm{C}$, after a half hour of a $1 \mathrm{KV}$ constant voltage pre-run. At least $0.4 \mathrm{KV}$-Hour pre-run procedure is essentially required to separate the salt line from DNA signals near the primer band.

\section{RESULTS AND CONCLUSION}

Figure 3 shows a portion of the obtained raw signal. In this case, around 471 mer nucleotide peaks were separated.

Figure 3 Portion of Raw Signal Obtained from $0.5 \mu \mathrm{g}$ (loaded) of M13mp18 Single Stranded DNA
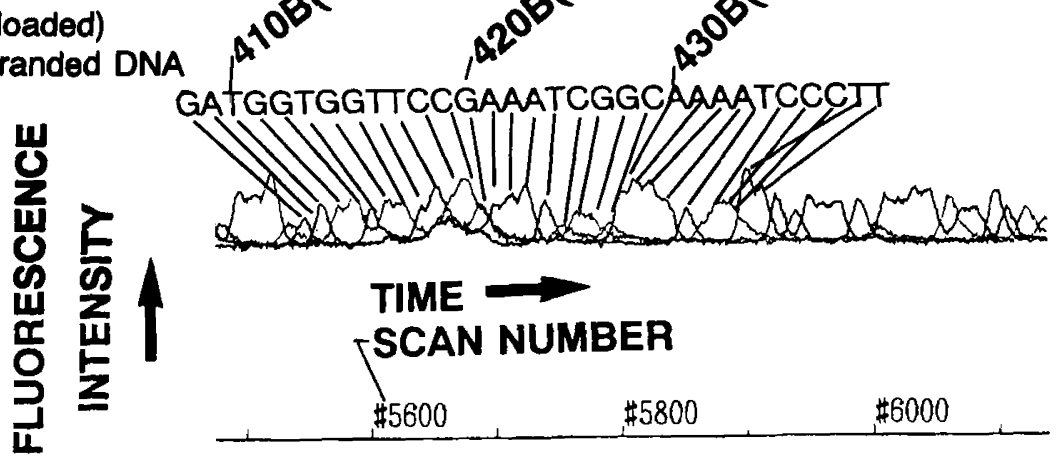

TIME SCAN NUMBER 
Figure 4 shows an example of analyzed data and sequence. A further homology analysis of the obtained sequence showed no error up to 507 bases.

Figure 4 Portions of Analyzed Data and Obtained Sequence from 0.5 $\mu \mathrm{g}$ (loaded) of M13mp18 DNA
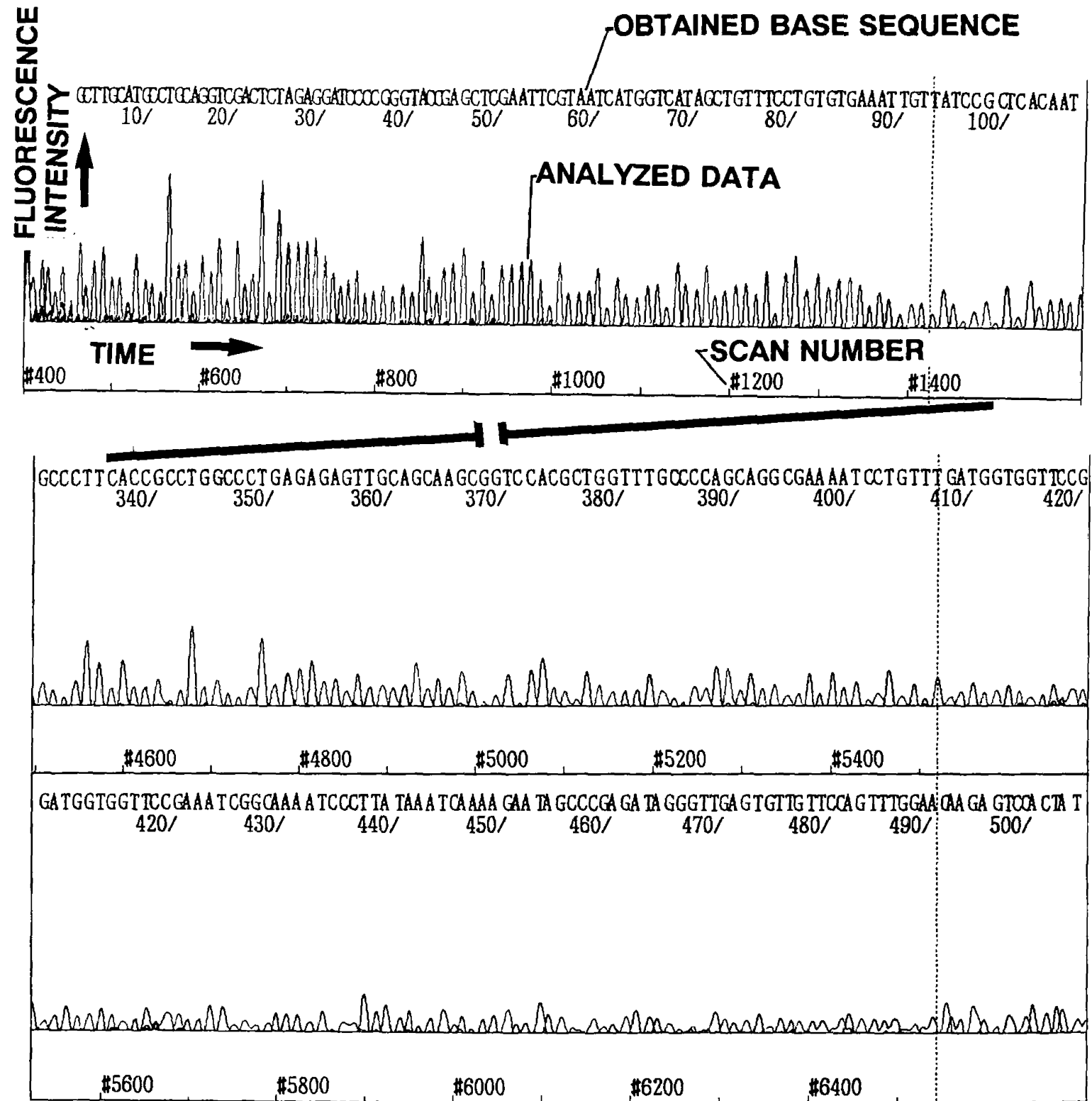

\section{ACKNOWLEDGEMENTS}

The authors are grateful to Dr Akio Yamane and Mr Shintaro Kawai of Wakunaga Pharmaceutical Company Limited, Hiroshima, Japan for giving us important chemicals including various fluorescent primers.

\section{REFERENCES}

1. Smith,M.L., Sander,J.Z., Kaiser,R.J., Hughes,P.. Dodd,C., Connell,C.R.,Heiner,C., Kent,S.B.H, and Hood,L.E., (1986) Nature 321, 674-679

2. Prober,J.M., Trainor,G.L., Dam,R.J., Hobbs,W.F., Robertson,C.W.,Zagursky,J.R., Cocuzza,A.J., Jensen,M.A., and Braumeister,K., (1987) Science 238, 336-341

3. Ansorge,W., Sproat,B.S., Stegemann,J., and Schwager,C. (1986) J.Biochem.Biophys.Methods 13, 315-323

4. Kambara,H., Nishikawa,T., Katayama,Y., and Yamaguchi,T., (1988) Bio/Technology 6, 816-821

5. Sanger,F., Nicklen,S. and Coulson,A.R., (1977) Proc.Natt.Acad.Sci.USA 74, 5463-5467 